\title{
SIMPLE SUFFICIENT CONDITIONS FOR BOUNDED TURNING
}

\author{
N. TUNESKI, M. DARUS, AND E. GELOVA
}

\begin{abstract}
Let $f$ be an analytic function in the open unit disk normalized such that $f(0)=f^{\prime}(0)-1=0$. In this paper the modulus and the real part of the linear combination of $f^{\prime}(z)$ and $f(z) / z$ is studied and conditions when $f$ is with bounded turning are obtained.
\end{abstract}

\section{INTRODUCTION AND PRELIMINARIES}

Let $\mathcal{A}$ denote the class of analytic functions in the unit disk $\mathbb{D}=\{z:|z|<1\}$ that are normalized such that $f(0)=f^{\prime}(0)-1=0$, i.e. $f(z)=z+a_{2} z^{2}+a_{3} z^{3}+\cdots$.

Function $f \in \mathcal{A}$ is in the class of starlike functions, $S^{*}$, if and only if

$$
\operatorname{Re}\left[\frac{z f^{\prime}(z)}{f(z)}\right]>0, \quad z \in \mathbb{D} .
$$

Such functions are univalent and their geometric characterization (which motivates the name of the class) is that they map the unit disk onto a starlike region, i.e. if $\omega \in f(\mathbb{D})$ then $t \omega \in f(\mathbb{D})$ for all $t \in[0,1]$.

Another well known class of univalent functions is the class of functions with bounded turning,

$$
R=\left\{f \in \mathcal{A}: \operatorname{Re} f^{\prime}(z)>0, z \in \mathbb{D}\right\} .
$$

Here also, the name of class follows from its geometric characterization, i.e. from the fact that $\operatorname{Re} f^{\prime}(z)>0$ is equivalent with $\left|\arg f^{\prime}(z)\right|<\pi / 2$ and $\arg f^{\prime}(z)$ is the angle of rotation of the image of a line segment from $z$ under the mapping $f$.

More details on these classes can be found in [2]. One of the main results concerning them is due to Krzyz ([7]), claiming that $S^{*}$ does not contain $R$ and $R$ does not contain $S^{*}$. This makes class $R$ interesting and lots of research is dedicated to it. Some references in that direction are [6] - [9].

In this paper we will study the linear combination of two simple expressions, $f^{\prime}(z)$ and $f(z) / z$, i.e. we will study the modulus and the real part of

$$
\alpha \cdot f^{\prime}(z)+\beta \cdot \frac{f(z)}{z}
$$

2010 Mathematics Subject Classification. 30C45.

Key words and phrases. analytic function, bounded turning, criteria, real part, modulus. 
and receive criteria for a function $f \in \mathcal{A}$ to be of bounded turning. For that purpose we will use a method from the theory of differential subordinations. Valuable references on this topic are [1] and [3].

First we introduce subordination. Let $f, g \in \mathcal{A}$. Then we say that $f(z)$ is $s u b$ ordinate to $g(z)$, and write $f(z) \prec g(z)$, if there exists a function $\omega(z)$, analytic in the unit disc $\mathbb{D}$, such that $\omega(0)=0,|\omega(z)|<1$ and $f(z)=g(\omega(z))$ for all $z \in \mathbb{D}$. Specially, if $g(z)$ is univalent in $\mathbb{D}$ then $f(z) \prec g(z)$ if and only if $f(0)=g(0)$ and $f(\mathbb{D}) \subseteq g(\mathbb{D})$.

For obtaining the main result we will use the method of differential subordinations. The general theory of differential subordinations, as well as the theory of first-order differential subordinations, was introduced by Miller and Mocanu in [4] and [5]. Namely, if $\phi: \mathbb{C}^{2} \rightarrow \mathbb{C}$ (where $\mathbb{C}$ is the complex plane) is analytic in a domain $D$, if $h(z)$ is univalent in $\mathbb{D}$, and if $p(z)$ is analytic in $\mathbb{D}$ with $\left(p(z), z p^{\prime}(z)\right) \in D$ when $z \in \mathbb{D}$, then $p(z)$ is said to satisfy a first-order differential subordination if

$$
\phi\left(p(z), z p^{\prime}(z)\right) \prec h(z) .
$$

The univalent function $q(z)$ is said to be a dominant of the differential subordination (2) if $p(z) \prec q(z)$ for all $p(z)$ satisfying (2). If $\widetilde{q}(z)$ is a dominant of (2) and $\widetilde{q}(z) \prec q(z)$ for all dominants of (2), then we say that $\widetilde{q}(z)$ is the best dominant of the differential subordination (2).

From the theory of first-order differential subordinations we will make use of the following lemma.

Lemma 1 ([5]). Let $q(z)$ be univalent in the unit disk $\mathbb{D}$, and let $\theta(\omega)$ and $\phi(\omega)$ be analytic in a domain $D$ containing $q(\mathbb{D})$, with $\phi(\omega) \neq 0$ when $\omega \in q(\mathbb{D})$. Set $Q(z)=z q^{\prime}(z) \phi(q(z)), h(z)=\theta(q(z))+Q(z)$, and suppose that

i) $Q(z)$ is starlike in the unit disk $\mathbb{D}$; and

ii) $\operatorname{Re} \frac{z h^{\prime}(z)}{Q(z)}=\operatorname{Re}\left\{\frac{\theta^{\prime}(q(z))}{\phi(q(z))}+\frac{z Q^{\prime}(z)}{Q(z)}\right\}>0, z \in \mathbb{D}$.

If $p(z)$ is analytic in $\mathbb{D}$, with $p(0)=q(0), p(\mathbb{D}) \subseteq D$ and

$$
\theta(p(z))+z p^{\prime}(z) \phi(p(z)) \prec \theta(q(z))+z q^{\prime}(z) \phi(q(z))=h(z)
$$

then $p(z) \prec q(z)$, and $q(z)$ is the best dominant of (3).

Now, using Lemma 1 we will prove the following result.

Lemma 2. Let $f \in \mathcal{A}$ and $\alpha, \beta \in \mathbb{C} \backslash\{0\}$ be such that $\alpha+\beta=0$ or $\alpha+\beta=1$. Also, let $q(z)$ be univalent in the unit disk $\mathbb{D}$ satisfying $q(0)=0$ and

$$
\operatorname{Re}\left[1+\frac{z q^{\prime \prime}(z)}{q^{\prime}(z)}\right]>0, \quad z \in \mathbb{D} \text {. }
$$


Additionally, $\operatorname{Re} \frac{1}{\alpha}>-1$ and

$$
\operatorname{Re}\left[1+\frac{z q^{\prime \prime}(z)}{q^{\prime}(z)}\right]>-\operatorname{Re} \frac{1}{\alpha}, \quad z \in \mathbb{D},
$$

in the case when $\alpha+\beta=1$. If

$$
\alpha \cdot f^{\prime}(z)+\beta \cdot \frac{f(z)}{z} \prec(\alpha+\beta) \cdot[q(z)+1]+\alpha z q^{\prime}(z) \equiv h(z)
$$

then $\frac{f(z)}{z}-1 \prec q(z)$, and $q(z)$ is the best dominant of $(6)$.

Proof. Functions $\theta(\omega)=(\alpha+\beta) \cdot(\omega+1)$ and $\phi(\omega)=\alpha$ are analytic in a domain $D=\mathbb{C}$ which contains $q(\mathbb{D})$ and $\phi(\omega) \neq 0$ when $\omega \in q(\mathbb{D})$. Further, $Q(z)=$ $z q^{\prime}(z) \phi(q(z))=\alpha z q^{\prime}(z)$ is starlike since

$$
\operatorname{Re} \frac{z Q^{\prime}(z)}{Q(z)}=\operatorname{Re}\left[1+\frac{z q^{\prime \prime}(z)}{q^{\prime}(z)}\right]>0, \quad z \in \mathbb{D}
$$

and for the function $h(z)=\theta(q(z))+Q(z)=Q(z)$ we have

$$
\operatorname{Re} \frac{z h^{\prime}(z)}{Q(z)}=\operatorname{Re}\left[1+\frac{\alpha+\beta}{\alpha}+\frac{z q^{\prime \prime}(z)}{q^{\prime}(z)}\right]>0, \quad z \in \mathbb{D},
$$

for $\alpha+\beta=0$ due to (4) and for $\alpha+\beta=1$ due to (5).

Now, let choose $p(z)=\frac{f(z)}{z}-1$ which is analytic in $\mathbb{D}, p(0)=q(0)=0$ and $p(\mathbb{D}) \subseteq$ $D=\mathbb{C}$. Finally, having in mind that subordinations (3) and (6) are equivalent, from Lemma 1 we receive the conclusions of Lemma 2.

\section{Results over the modulus of (1)}

In this section we will study the modulus of (1) and receive conclusions that will lead to criteria for a function $f$ to be in the class $R$.

Theorem 1. Let $f \in \mathcal{A}, \mu>0$ and $\alpha, \beta \in \mathbb{C} \backslash\{0\}$ be such that $\alpha+\beta=0$ or $\alpha+\beta=1$. Also, let $\operatorname{Re} \frac{1}{\alpha}>-1$ in the case when $\alpha+\beta=1$. If

$$
\left|\alpha \cdot f^{\prime}(z)+\beta \cdot \frac{f(z)}{z}-(\alpha+\beta)\right|<\delta \equiv\left\{\begin{array}{cc}
\mu \cdot|\alpha|, & \alpha+\beta=0 \\
\mu \cdot|1+\alpha|, & \alpha+\beta=1
\end{array},\right.
$$

for all $z \in \mathbb{D}$, then

$$
\left|\frac{f(z)}{z}-1\right|<\mu, \quad z \in \mathbb{D} .
$$

This implication is sharp, i.e., in the inequality (8), $\mu$ can not be replaced by a smaller number so that the implication holds. Also,

$$
\left|f^{\prime}(z)-1\right|<\lambda \equiv\left\{\begin{array}{cc}
2 \mu, & \alpha+\beta=0 \\
\mu \cdot\left(\left|1+\frac{1}{\alpha}\right|+\left|1-\frac{1}{\alpha}\right|\right), & \alpha+\beta=1
\end{array}\right\}, \quad z \in \mathbb{D}
$$

This implication is also sharp, i.e., $\lambda$ can not be replaced by a smaller number so that the implication holds, if 
(i) $\alpha+\beta=0$; or

(ii) $\alpha+\beta=1$ and $\left|1+\frac{1}{\alpha}\right|+\left|1-\frac{1}{\alpha}\right|=2$.

Additionally, if $\mu \leq \frac{1}{2}$ for $\alpha+\beta=0$ or $\left|1+\frac{1}{\alpha}\right|+\left|1-\frac{1}{\alpha}\right| \leq \frac{1}{\mu}$ for $\alpha+\beta=1$ then $f \in R$.

Proof. Choosing $q(z)=\mu z$ we have $1+\frac{z q^{\prime \prime}(z)}{q^{\prime}(z)}=1$, meaning that (4) and (5) form Lemma 2 hold. Further, for the function $h(z)$ defined in (6) we have

$$
h(z)=\alpha+\beta+\mu z(2 \alpha+\beta),
$$

meaning that subordination (6) is equivalent to

$$
\left|\alpha \cdot f^{\prime}(z)+\beta \cdot \frac{f(z)}{z}-(\alpha+\beta)\right|<\mu \cdot|2 \alpha+\beta|=\delta, \quad z \in \mathbb{D},
$$

i.e. to (7). Therefore, (8) follows directly from Lemma 2 and the definition of subordination.

Further, for all $z \in \mathbb{D}$,

$$
\left|\alpha \cdot f^{\prime}(z)+\beta \cdot \frac{f(z)}{z}-(\alpha+\beta)\right|=\left|\alpha \cdot\left[f^{\prime}(z)-1\right]+\beta \cdot\left[\frac{f(z)}{z}-1\right]\right|
$$

and

$$
\begin{aligned}
|\alpha| \cdot\left|f^{\prime}(z)-1\right| & \leq\left|\alpha \cdot\left[f^{\prime}(z)-1\right]+\beta \cdot\left[\frac{f(z)}{z}-1\right]\right|+\left|\beta \cdot\left[\frac{f(z)}{z}-1\right]\right| \\
& <\delta+|\beta| \cdot \mu=|\alpha| \cdot \lambda,
\end{aligned}
$$

since $\left|w_{1}\right| \leq\left|w_{1}+w_{2}\right|+\left|w_{2}\right|$. Therefore, the implication of this corollary holds.

Both implication are sharp as the function $f_{*}(z)=z+\mu z^{2}$ shows, since

$$
\begin{gathered}
\left|\alpha \cdot f_{*}^{\prime}(z)+\beta \cdot \frac{f_{*}(z)}{z}-(\alpha+\beta)\right|=\mu \cdot|2 \alpha+\beta| \cdot|z|=\delta \cdot|z|, \quad z \in \mathbb{D}, \\
\left|\frac{f_{*}(z)}{z}-1\right|=\mu \cdot|z|, \quad z \in \mathbb{D}, \\
\left|f_{*}^{\prime}(z)-1\right|=2 \cdot \mu \cdot|z|, \quad z \in \mathbb{D},
\end{gathered}
$$

and $2 \mu=\lambda$ if (i) or (ii) hold.

\section{Results over the real part of (1)}

In this section we will study the real part of the expression (1) and receive criteria over it that will embed a function $f \in \mathcal{A}$ in the class $R$.

Theorem 2. Let $f \in \mathcal{A}, \mu>0$ and $\alpha, \beta \in \mathbb{C} \backslash\{0\}$ be such that $\alpha+\beta=0$ or $\alpha+\beta=1$. Also, let $\operatorname{Re} \frac{1}{\alpha}>0$ in the case when $\alpha+\beta=1$. If

$$
\alpha \cdot f^{\prime}(z)+\beta \cdot \frac{f(z)}{z} \prec(\alpha+\beta)\left(1+\frac{2 \mu z}{1-z}\right)+\frac{2 \alpha \mu z}{(1-z)^{2}} \equiv h_{2}(z)
$$


then

$$
\operatorname{Re}\left[\frac{f(z)}{z}\right]>1-\mu, \quad z \in \mathbb{D} .
$$

This implication is sharp, i.e., in the inequality (10), $\mu$ can not be replaced by a bigger number so that the implication holds.

Proof. The implication of this theorem follows directly from Lemma 2 for $q(z)=$ $\frac{2 \mu z}{1-z}$. Condition $\operatorname{Re} \frac{1}{\alpha}>0$ stands in stead of $\operatorname{Re} \frac{1}{\alpha}>-1$ in order (5) to hold. The result is sharp due to the function $f_{*}(z)=z+z \cdot q(z)$ such that

$$
\alpha \cdot f_{*}^{\prime}(z)+\beta \cdot \frac{f_{*}(z)}{z}=(\alpha+\beta)\left(1+\frac{2 \mu z}{1-z}\right)+\frac{2 \alpha \mu z}{(1-z)^{2}}
$$

and $\operatorname{Re} \frac{f(z)}{z}=1-\mu$ for $z=-1$.

In the case when $\alpha+\beta=1$ we receive the following corollary.

Corollary 1. Let $f \in \mathcal{A}, \alpha>0$ and $\mu>0$. If

$$
\operatorname{Re}\left[\alpha \cdot f^{\prime}(z)+(1-\alpha) \cdot \frac{f(z)}{z}\right]>1-\mu \cdot\left(1+\frac{\alpha}{2}\right), \quad z \in \mathbb{D},
$$

then

If, additionally,

$$
\operatorname{Re}\left[\frac{f(z)}{z}\right]>1-\mu, \quad z \in \mathbb{D} \text {. }
$$

(i) $\alpha>1$ and $\mu \leq 1$; or

(ii) $\alpha<1$ and $\mu \geq 1$;

then

$$
\operatorname{Re} f^{\prime}(z)>1-\frac{3}{2} \cdot \mu, \quad z \in \mathbb{D} \text {. }
$$

These results are sharp.

Proof. Let $\alpha+\beta=1$. So, for the function $h_{2}$ defined in (9) we have

$h_{2}(0)=1$ and

$$
h_{2}(z)=1+\frac{2 \mu z}{1-z}+\frac{2 \alpha \mu z}{(1-z)^{2}}
$$

$$
h_{2}\left(e^{i \theta}\right)=1-\frac{\mu \alpha}{2}\left(1+t^{2}\right)-\mu+\mu t i,
$$

where $t=\operatorname{ctg}(\theta / 2)$. Therefore,

$$
X=\operatorname{Re} h\left(e^{i \theta}\right)=1-\mu\left(\frac{\alpha}{2}+1\right)-\frac{\alpha}{2 \mu} \cdot Y^{2},
$$

where

$$
Y=\operatorname{Im} h\left(e^{i \theta}\right)=\mu t
$$


attains all real numbers. This leads to

$$
h_{2}\left(e^{i \theta}\right)=\left\{x+i y: x=1-\mu\left(1+\frac{\alpha}{2}\right)-\frac{\alpha}{2 \mu} \cdot y^{2}, y \in \mathbb{R}\right\} .
$$

From here, having in mind the definition of subordination, the inequality (11) and the fact that

$$
\left\{x+i y: x>1-\mu\left(1+\frac{\alpha}{2}\right), y \in \mathbb{R}\right\} \subseteq h_{2}(\mathbb{D}),
$$

we receive subordination (9). Therefore, from Theorem 2 follows

$$
\operatorname{Re}\left[\frac{f(z)}{z}\right]>1-\mu, \quad z \in \mathbb{D} \text {. }
$$

Further, in the case when (i) or (ii) holds we have

$$
\begin{aligned}
\operatorname{Re} f^{\prime}(z) & =\frac{1}{\alpha} \cdot\left\{\operatorname{Re}\left[\alpha \cdot f^{\prime}(z)+(1-\alpha) \cdot \frac{f(z)}{z}\right]-(1-\alpha) \cdot \operatorname{Re}\left[\frac{f(z)}{z}\right]\right\} \\
& >\frac{1}{\alpha} \cdot\left[1-\mu\left(1+\frac{\alpha}{2}\right)-(1-\alpha)(1-\mu)\right]=1-\frac{3}{2} \cdot \mu
\end{aligned}
$$

for all $z \in \mathbb{D}$.

The results are sharp due to the function $f_{*}(z)=z+\frac{2 \mu z^{2}}{1-z}$ such that $f_{*}(z) / z=$ $1+\frac{2 \mu z}{1-z} \equiv g(z), g(\mathbb{D})=\{x+i y: x>1-\mu, y \in \mathbb{R}\}$,

$$
\alpha \cdot f_{*}^{\prime}(z)+(1-\alpha) \cdot \frac{f_{*}(z)}{z}=h_{2}(z)
$$

and

$$
\operatorname{Re} f_{*}^{\prime}(z)=\operatorname{Re} h_{2}(z)=1-\frac{3}{2} \cdot \mu \text { for } z=-1
$$

In a similar way as in Corollary 1 , for the case $\alpha=-\beta=1$ we receive

Corollary 2. Let $f \in \mathcal{A}$ and $\mu>0$. If

$$
\operatorname{Re}\left[f^{\prime}(z)-\frac{f(z)}{z}\right]>-\frac{\mu}{2}, \quad z \in \mathbb{D},
$$

then $\operatorname{Re}\left[\frac{f(z)}{z}\right]>1-\mu, z \in \mathbb{D}$, and $\operatorname{Re} f^{\prime}(z)>1-\frac{3}{2} \cdot \mu, z \in \mathbb{D}$. If, additionally, $\mu \leq \frac{2}{3}$, then $\operatorname{Re} f^{\prime}(z)>0, z \in \mathbb{D}$, i.e. $f \in R$. Both implications are sharp.

\section{EXAmples}

The following example exhibits some concrete conclusions that can be obtained from the results of the previous sections by specifying the values $\alpha, \beta$ and $\mu$.

Example 1. Let $f \in \mathcal{A}$.

(i) If $\left|f^{\prime}(z)-\frac{f(z)}{z}\right|<\frac{1}{2}(z \in \mathbb{D})$ then $\left|f^{\prime}(z)-1\right|<1(z \in \mathbb{D})$ and $f \in R$. ( $\alpha=-\beta=1$ and $\mu=\frac{1}{2}$ in Theorem 1 ); 
(ii) If $\left|f^{\prime}(z)+\frac{f(z)}{z}-2\right|<1(z \in \mathbb{D})$ then $\left|f^{\prime}(z)-1\right|<1(z \in \mathbb{D})$ and $f \in R$. ( $\alpha=\beta=\frac{1}{2}$ and $\mu=\frac{1}{4}$ in Theorem 1$)$;

(iii) If $\alpha>0$ and $\operatorname{Re}\left[\alpha \cdot f^{\prime}(z)+(1-\alpha) \cdot \frac{f(z)}{z}\right]>-\frac{\alpha}{2}(z \in \mathbb{D})$ then $\operatorname{Re}\left[\frac{f(z)}{z}\right]>$ $0(z \in \mathbb{D})$ and $\operatorname{Re} f^{\prime}(z)>-1 / 2(z \in \mathbb{D}) . \quad(\mu=1$ in Corollary 1$) ;$

(iv) If $\operatorname{Re}\left[f^{\prime}(z)+\frac{f(z)}{z}\right]>-\frac{1}{2}(z \in \mathbb{D})$ then $\operatorname{Re}\left[\frac{f(z)}{z}\right]>0(z \in \mathbb{D})$ and $\operatorname{Re} f^{\prime}(z)>-1 / 2(z \in \mathbb{D}) . \quad(\alpha=1 / 2$ and $\mu=1$ in Corollary 1$) ;$

(v) If $\operatorname{Re}\left[f^{\prime}(z)-\frac{f(z)}{z}\right]>-\frac{1}{3}(z \in \mathbb{D})$ then $\operatorname{Re} f^{\prime}(z)>0(z \in \mathbb{D})$ and $f \in R$. ( $\mu=\frac{2}{3}$ in Corollary 2);

Remark 1. It is worth noting that in part (iii) of the previous example, the conclusion does not depend on $\alpha$.

\section{REFERENCES}

[1] Bulboaca T., Differential subordinations and superordinations. New results, House of Science Book Publ., Cluj-Napoca, 2005.

[2] Duren P.L., Univalent functions, Springer-Verlag,1983.

[3] Miller S.S., Mocanu P.T., Differential subordinations, Theory and Applications, Marcel Dekker, New York-Basel (2000).

[4] Miller S.S., Mocanu P.T., Differential subordinations and univalent functions, Michigan Math. J. 28 (1981), 157-171.

[5] Miller S.S., Mocanu P.T., On some classes of first-order differential subordinations, Michigan Math. J. 32 (1985), 185-195.

[6] Ibrahim R.W., Darus M., Extremal bounds for functions of bounded turning, Int. Math. Forum 6 (33) (2011), 1623-1630.

[7] Krzyz J., A counter example concerning univalent functions, Mat. Fiz. Chem. 2 (1962), 57-58.

[8] Tuneski N., Some simple sufficient conditions for starlikeness and convexity, Appl. Math. Lett. 22 (2009), 693-697.

[9] Tuneski N., Obradovic M., Some properties of certain expression of analyti functions, Computers and Mathematics with Applications 62 (2011), 3438-3445.

Faculty of Mechanical Engineering, Ss. Cyril and Methodius University in Skopje, Karpoš II B.B., 1000 Skopje, Republic of Macedonia.

E-mail address: nikola.tuneski@mf.edu.mk

School of Mathematical Sciences,Faculty of science and Technology Universiti Kebangsaan Malaysia, Bangi 43600, Selangor Darul Ehsan, Malaysia

E-mail address: maslina@ukm.my

University Goce Delcev, Štip, Republic of Macedonia

E-mail address: gelovae@yahoo.com 